\title{
Evolution of Population with Interaction between Neighbours
}

\author{
Maria Magdoń-Maksymowicz ${ }^{1}$ and Andrzej Z. Maksymowicz ${ }^{2}$ \\ 1 Department of Mathematical Statistics \\ AR-Agricultural University \\ Al. Mickiewicza 21 \\ 31-120 Krakow, Poland \\ rrmagdon@cyf-kr.edu.pl \\ 2 Faculty of Physics and Nuclear Techniques \\ AGH-University of Science and Technology \\ Al. Mickiewicza 30 \\ 30-059 Krakow, Poland
}

\begin{abstract}
Dynamic of population growth on two-dimensional lattice $N_{x} \times N_{y}=1000 \times 1000$ is simulated with rules that involve interaction between neighbours. Biological aging is described by a systematic decrease with each time step of parameter $g(i)$, a measure of the health condition for each individual $i$. When $g$ drops down below a minimum value, the death of the item occurs. The death toll is also caused by the Verhulst factor which accounts for the limited living space. Newly borns inherit $g$ from parent's, plus some extra value to help them to start the life game. This basic rule is enriched by (a) some corrections of the parameter $g(i)$ according to its current values among the nearest and next nearest neighbours, and/or (b) the possible limitation of newborns to be placed in the close neighbourhood of parents. We discuss influence of the birth rate on the size of the final population and its age distributions, then $g-g$ correlations $c$ of the health condition $g$ on the nearest neighbours. Main conclusion is that interaction between neighbours may play important role modifying the population characteristics, also the restrictions on whether the babies are bound to stay with parents or not yield different results.
\end{abstract}

\section{Introduction}

Population evolution is governed by a set of rules which describe how the state of population $n(t)$ at time $t$ yields the population $n(t+1)$ at the next time-step $t+1$. The rules must account for the elimination mechanism of items and also for a new items entering the society. In equilibrium, the number of deaths $\Delta n$ must be balanced by same number of birth, on the average. Probabilistic approach to the rules may lead to either an analytical description of continuous time sequence or to iterative procedures suitable for computer simulations. Among them, cellular automata may be considered as the tool which we apply in this work. In the simplest model we assume death caused by 
the limited environmental capacity $N$, so that in each time step we eliminate a fraction $n(t) / N$ of population, known as the Verhulst factor [1. The deaths are compensated by introducing a fraction $B$ of the remaining individuals, perhaps with the limitation on the minimum reproduction age $R$. The net balance predicts the equilibrium $n$ and also age distribution $n(a)$. In a more realistic approach we need to account for different deaths mechanisms. Among them, the most often named are bad mutations, oxygen radicals and longevity genes. In the Penna model 2], the population evolution is seen as controled by the accumulation of bad mutations, passed over to offsprings which also may catch additional mutations. The mutations for each individual $(i)$ are represented by bit '1' in the genome - a computer word. The bad mutations are not harmful until they are activated. This takes place when we disclose the next bit position at each time step, and the sum of already active mutations is then compared against a threshold value $(T)$. The individual dies on reaching this value. The child genome is inherited from its parent (cloning in the asexual version of the Penna model) with eventually additional mutations spelled at randomly chosen bits of the genome. This Penna model is perhaps the most popular evolution model which simulates biological aging and genetic death (see [3] for review).

Both the rate of born babies, as well as possible deaths scenarios are far more complex then the very simple model presented in this paper. The aim of this work is to test how the very local interactions between neighbouring items may be important. We intend to account for a socializing factor in local communities such as a family or close neighbours. Very often members of such small communities create their own rules and influence each other. This influence may be incorporated into the more general rules that control all population. The first simple idea is to try to adjust parameter $g(i)$, a sort of a fitness parameter of each member $i$, so that better neighbour improves our condition, and poorer neighbour makes us less fit. In the $2 \mathrm{D}$ lattice model, each item occupies a cell and state of all items in the neighbouring cells has some effect on the cell in consideration.

\section{Model}

The population $n$ is displaced on a two-dimensional $N=N_{x} \times N_{y}=1000 \times$ 1000 lattice, each $i$-th item occupying one cell. Individuals are characterized by a goodness parameter $g(i)$ ranging from a minimum value $g_{\text {min }}$, below which the item is considered dead, up to a certain $g_{\max }$ value. At each time step of simulation the age $a(i)$ is icreased by one and the goodness $g$ is diminished by $\Delta g$. The Initial population $n(0)<N$ at time $t=0$ is randomly spread all over the lattice. At each simulation step $t \rightarrow t+1$, all items are scanned. For each item we apply the following rules.

- The item is eliminated with probability $n(t) / N$, the Verhulst factor.

- If it survives and its age $a$ is at least the minimum reproduction age $R$, $B$ babies may be born. That is, only when randomly chosen cell where we intend to place the newborn is empty, the actual number of offsprings is $B$; 


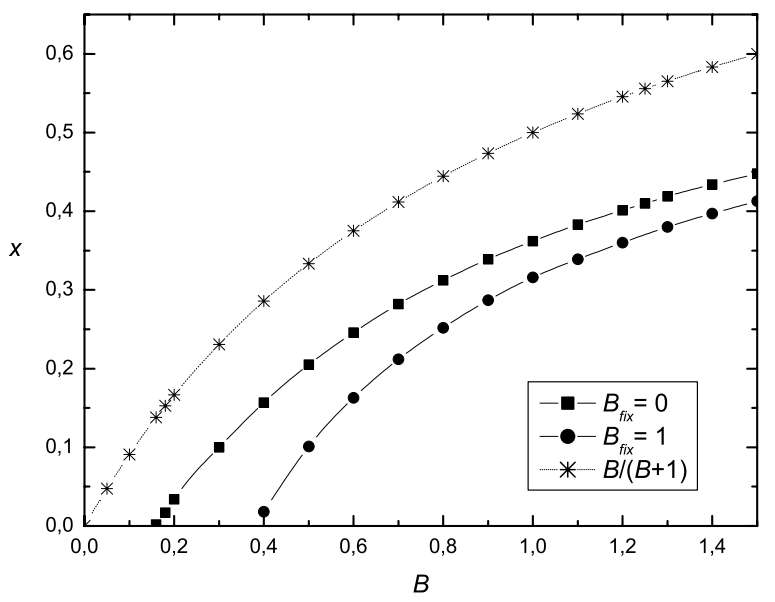

Fig. 1. Normalized population $x$ as a function of the birth rate $B$. The top curve is the logistic case. Results marked by squares are obtained for the newborns randomly located on the lattice, $\left(B_{f i x}=0\right)$. Circles correspond to the case when babies are located in the neighbourhood of parents, $\left(B_{f i x}=1\right)$

otherwise the number is smaller or even zero if all proposed cells happen to be full. In this asexual version, the baby at age $a=0$ gets initial $g$ of the parent, plus some extra $\delta$ value as a beneficial amount.

- Looking around, the $i$-th item's $g(i)$ is increased (by $g_{n n}=2$ in case of any of the four nearest neighbours and by $g_{n n n}=1$ for the 4 next nearest neighbours) if the neighbour has larger $g$. If the neighbour has smaller $g$, $g(i)$ is decreased by $g_{n n}$ and/or $g_{n n n}$, respectively. In other words, the social interaction makes the local society tends to become more uniform.

- Elimination caused by poor health condition takes now place for the weakest members with $g<g_{\text {min }}$.

- The last step which completes the transition from $t$ to $t+1$ era is the update, the age $a$ increases by one and goodness $g$ drops by $\Delta g$.

It may be noticed some similarities with the Penna model. The systematic decrease in $g$ has similar effect as the activation of bad mutations in the Penna model. The extra amount $\delta$ of $g$ value inherited from parent is equivalent to resetting the baby's counter of the active bad mutations to zero.

\section{Results and Discussion}

We used for the minimum goodness factor $g_{\text {min }}=90$, below which items are eliminated. Biological aging makes $g(i)$ drop down at rate of $\Delta g=1$ per time step. The follow-the-neighbour shifts of current $g(i)$ 's are $g_{n n}=2$ and $g_{n n n}=1$, 
or none if we switched off this interaction. The minimum reproduction age was assumed $R=0$. The babies gain credit $\delta=2$, so that $g$ (baby) $=g$ (parent) $+\delta$ for newborns.

Figure 1 shows population size vs birth rate. The logistic game, with the Verhulst factor as the only possible elimination mechanism of an item, offers the most densely populated case. The equilibrium population shows no critical birth rate $B_{c}$ below which the population is extinct. In the described cellular automata model, the death also occurs for other reasons - below the minimum health condition - and so population $x$ is then smaller.

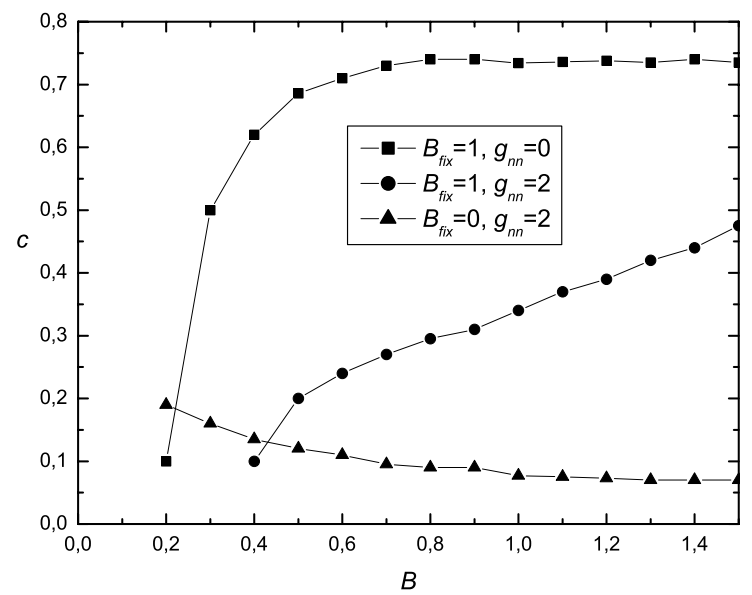

Fig. 2. Correlation $c$ of individuals' health condition against the birth rate $B$. Results marked by squares or circles are obtained for the case of babies kept close to parents; circles correspond to the follow-the-neighbour-condition rule, while results marked by squares ignore the neighbours. The triangles are for no babies spread over the whole lattice, yet we still mind the neighbours

In particular, we have nonzero $B_{c}$. The two lower curves are obtained for different bounds imposed on the available living space by the two facts. Firstly, within the lattice cell approach, each item is subject to the (Pauli) exclusion principle that any cell cannot be occupied by more then one individual. So the effective space is not the lattice $N_{x} \times N_{y}=1000 \times 1000$ size yet it is smaller by factor $(1-x)$. Secondly, the rule of an offspring to be kept in close vicinity of the parent diminishes further the effective space, the lowest curve in Fig. 1]

Results shown on Fig. 2 is the summary of how far the health conditions of the nearest neighbours are correlated. The invisible horizontal $c=0$ line of uncorrelated values is due to the obvious case of babies placed on the whole 


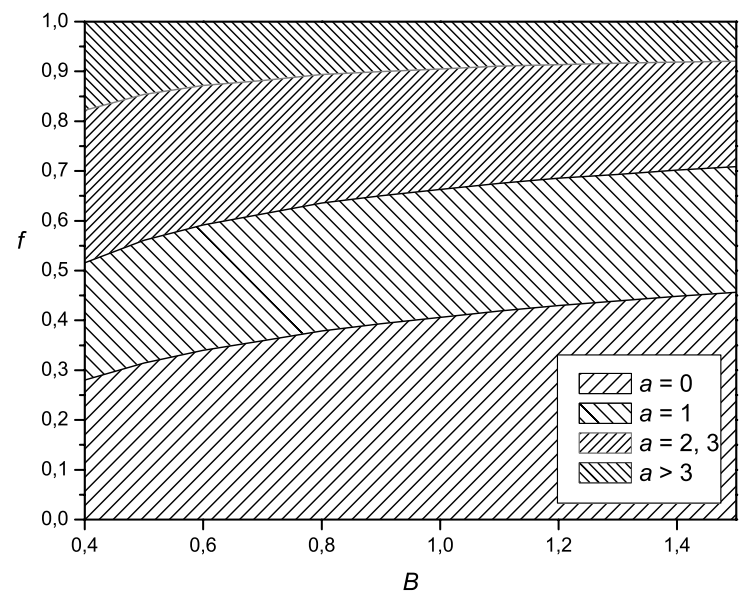

Fig. 3. Age distribution $f$ for babies $(a=0)$, young $(a=1)$, mature $(a=2,3)$ and old $(a>3)$ members of society, with the follow-the-neighbour-condition rule, against the birth rate. Babies are free to choose location

lattice and ignoring our local neighbours. The most striking effect is for babies kept close to parents, the squares in the figure, as the basic mechanism of cloning ensures offsprings become similar to parents. (This also creates a local environment which may differ from the overall environmental characteristics.) However, if we switch on interaction between neighbours, the effect is less correlated population. We may see neighbours and influence neighbours who do not belong to the family. And vice versa, the neighbours also affect us. As we said, if babies are placed close by, it plays dominant role for the correlation. The correlations are still present if we accept the influence of neighbours only, and let children to wander all around. This time correlations are relatively smaller and they drop down with the birth rate. This is expected since for larger $B$, the more randomly placed new items brings in more uncorrelated strangers to the local community, before they become assimilated after the many iteration steps.

The division line between different age groups increases monotically with birth rate $B$. This is so since for higher reproduction rate there is larger inflow of younger generation members. Also there is less room left for older generation from the point of view of limited environmental space. Figures 3 and 4 are obtained for the newly borned placed randomly or in vicinity of parents. It is seen some, yet not very drastic, influence of this on the age distribution of the population. For small birth rates, with no bonds on where the children go, about $17 \%$ of populations are seniors. This fraction diminishes to $10 \%$ for large $B$, according to the mentioned mechanism of repelling older members if we need to accomodate more youngsters. However, if children are forced do stay close by, the corresponding numbers are smaller: $11 \%$ and $6 \%$. And, simultaneolusly, the 


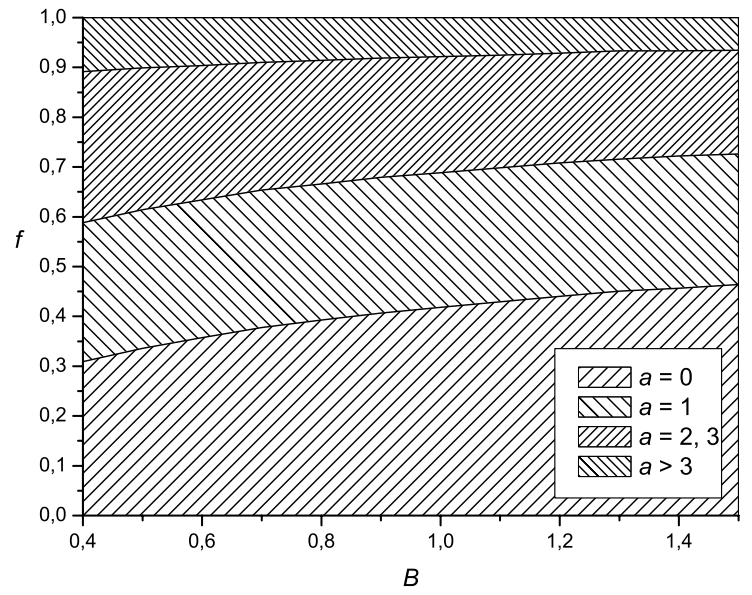

Fig. 4. Age distribution as in 3 yet this time babies are bound to stay near the parents

percentage of youngsters go up. In other words, the age distribution indicates that the whole population becomes younger. It may be interpreted as result of the benificial influence of babies, which gain the extra vital power on birth, and so improve the condition of parents if they are in the neighborhood. At the same time, the babies condition lessens. When growing up, on reaching the old age, they enter it with poorer health condition and so the death toll is higher. This is why percentage of the very old is smaller. However, we must be careful in interpretation of results for given birth rate $B$. Apart from general tendency, no direct quantitative comparison is possible as the efective birth rate $B_{\text {eff }}$ is always smaller then $B$ since no doubly occupied cells are allowed. The point is, this limitation is much more severe for the case when babies stay by parents, $B_{f i x}=1$.

For example, the age distribution $n(a)$ obtained from computer simulation strongly depends on the assumed restriction. The usually discussed characteristics is the mortality $q(a)=1-n(a+1) / n(a)$, which is a fraction of the population at age $a$ that will be eliminated before reaching the next $(a+1)$ age. The well known Gompertz law of exponential $q(a)$ dependence, $q(a) \sim e^{b \cdot a}$, predicts a straight line on a plot of $S(a)=\log q$ against age $a$. The results of simulations are presented on Fig. 5. It is seen that the linear dependence of $S(a)$ is not held. In human populations we observe a minimum and it is only for humans of age above 20 or 30 that the dependence is more or less linear; perhaps with exception of very old individuals when again the deviation from the Gompertz law may be observed. We do not intend to analyse the clear deviations from the Gompertz law, which may stem out from many reasons, and which is currently widely discussed in many publications, also in the already mentioned review 3 . 


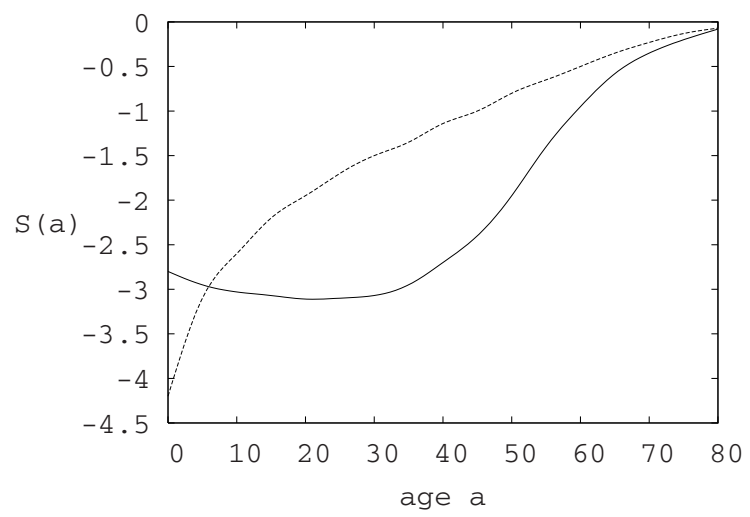

Fig. 5. Simulation results of age dependence of $S(a)=\log q$, the exponential Gompertz law of mortality $q(a)$ predicts a straight line. The line with a minimum is for whole lattice as possible location for newborns, the other line corresponds to offsprings located in close neighborhood to parent

In summary, we only conclude that by suitable choice of the model parameters, corresponding to different proposed mechanisms of evolution, we may try to reproduce population characteristics and try to interpret results in terms of appropriate rules controling the population dynamics.

Acknowledgements. The work was supported by a grant of the Agricultural University, Department of Mathematical Statistics, and by the University of Science and Technology, Faculty of Physics and Nuclear Techniques. Computer calculations were carried out at the Academic Computer Centre CYFRONETKRAKÓW on HP Exemplar S2000 machine.

\section{References}

1. D. Brown and P. Rolhery, Models in Biology: Mathematics, Statistics and Computing, Wiley, New York, 1993.

2. T. J. P. Penna, J. Stat. Phys. 78 (1995) 1629.

3. S. Moss de Oliveira, P. M. C. de Oliveira and D. Stauffer, Evolution, Money, War and Computers, Teubner, Stuttgart-Leipzig, 1999. 